\title{
The Incidence of Pulmonary Thromboembolism in Critically III Patients With COVID-19: A Systematic Review, Meta-Analysis and Meta-Regression of Observational Studies
}

Jun Jie Ng ( $\sim$ jun_jie_ng@nuhs.edu.sg )

National University Heart Centre, Singapore https://orcid.org/0000-0003-0779-8468

Zhen Chang Liang

National University Health System

Andrew MTL Choong

National University Health System

\section{Research}

Keywords: Coronavirus disease 2019, COVID-19, pulmonary embolism, pulmonary thromboembolism, intensive care unit, critical care

Posted Date: September 15th, 2020

DOI: https://doi.org/10.21203/rs.3.rs-74260/v1

License: @ (i) This work is licensed under a Creative Commons Attribution 4.0 International License. Read Full License 


\section{Abstract}

Purpose Coronavirus disease 2019 (COVID-19) infection is known to be associated with a hypercoagulable and prothrombotic state, especially in critically ill patients. Several observational studies have reported the incidence of thromboembolic events such as pulmonary thromboembolism (PTE). We performed a meta-analysis to estimate the weighted average incidence of PTE in critically ill COVID-19 patients who are admitted to the intensive care unit.

Methods We searched MEDLINE via PubMed, Embase and Web of Science for relevant studies from 31 December 2019 till 15 Aug 2020 onwards using the search terms "coronavirus", "COVID-19", "SARS-CoV-2", "2019-nCoV", "thrombus", "thrombo*", "embolus" and "emboli*”. We included prospective and retrospective observational studies that reported the incidence of PTE in critically ill COVID-19 patients who required treatment in the intensive care unit. We identified 14 studies after two phases of screening and extracted data related to study characteristics, patient demographics and the incidence of PTE. Risk of bias was assessed by using the ROBINS-I tool. Statistical analysis was performed with R 3.6.3.

Results We included 14 studies with a total of 1182 patients in this study. Almost $100 \%$ of patients in this meta-analysis received at least prophylactic anticoagulation. The weighted average incidence of PTE was $11.09 \%\left(95 \% \mathrm{Cl} 7.72 \%\right.$ to $15.69 \%, \mathrm{I}^{2}=78 \%$, Cochran's Q test $\left.\mathrm{P}<0.01\right)$. We performed univariate and multivariate meta-regression which identified the proportion of males as a significant source of heterogeneity $(P=0.03,95 \% \mathrm{Cl} 0.00$ to -0.09$)$

Conclusion This is the only study that had specifically reported the weighted average incidence of PTE in critically ill COVID-19 patients using meta-analytic techniques. The weighted average incidence of PTE remains high even after prophylactic anticoagulation. This study is limited by incomplete data from included studies. More studies are needed to determine the optimal anticoagulation strategy in critically ill COVID-19 patients.

\section{Background}

Since the declaration of a global pandemic by the World Health Organization on 11 March 2020, more than 25 million people have been diagnosed with coronavirus disease 2019 (COVID-19). Amongst them, over 800,000 people have died.[1] As we gradually understand more about the pathophysiology and clinical manifestations of COVID-19 infection, a few distinct themes have emerged. One of the more apparent themes is the hypercoagulable, prothrombotic state that critically-ill COVID-19 patients have an affinity for.[2] Early studies first reported autopsy findings of micro-thrombus within the pulmonary vasculature of deceased COVID-19 patients.[3] At the same time, other studies started reporting about abnormal coagulation parameters and elevated D-dimer levels in critically-ill COVID-19 patients. $[4,5]$ On the frontline, physicians treating critically-ill COVID-19 patients started noticing an increase in thromboembolic events and line thrombosis.[6] Cognizant of the thromboembolic phenomenon associated with COVID-19, several institutions have published observational studies that reported the incidence of thromboembolic events such as pulmonary thromboembolism (PTE). In this study, we aim to quantitatively synthesize available literature by using meta-analysis of proportions to estimate the weighted average incidence of PTE in critically-ill COVID-19 patients that are admitted to the intensive care unit (ICU).

\section{Methods}

\section{Study protocol}

We conducted this systematic review and meta-analysis following the Cochrane Handbook for Systematic Reviews of Interventions and reported it in accordance with the Preferred Reporting Items for Systematic Reviews and Meta-analyses (PRISMA) guidelines.[7, 8] We formulated the study protocol for this systematic review and meta-analysis in an apriori fashion and published it in PROSPERO (CRD42020188647).

\section{Search strategy}

We formulated the search strategy after discussion and consensus by all authors. The search strategy included various combinations and permutations of the following search terms: "coronavirus", "COVID-19", "SARS-CoV-2", "2019-nCoV, "thrombus", "thrombo*”, "embolus" and "emboli*”. We identified studies by conducting an exhaustive literature search using MEDLINE via PubMed, Embase and Web of Science. We modified the search syntax for compatibility as required for each database. We only included studies that were published after 31 December 2019, which corresponds to the date when Chinese officials first reported a cluster of patients diagnosed with pneumonia of unknown cause in Wuhan, Hubei Province to the World Health Organization.[9] We did not restrict language for the search. After eligible full-text studies were identified, we performed manual backward reference searching to ensure all relevant studies were included. We only included studies that were published in a peer-reviewed journal. We performed a repeat search on 1 September 2020 before submission to ensure no studies were missed.

\section{Eligibility criteria}

We included prospective and retrospective observational studies that reported the incidence of PTE in COVID-19 patients who were admitted to the ICU for treatment. We excluded individual case reports or case series on PTE in COVID-19 patients. We excluded studies that focused on reviewing all cross-sectional chest imaging, regardless of clinical indication, that had been performed for COVID-19 patients to determine the incidence of PTE in COVID-19 patients. We also excluded studies that had reported the incidence of all types of venous thromboembolism, without reporting separate incidences of pulmonary thromboembolism. Lastly, we excluded studies that were not published in peer-reviewed journals such as studies that are published in pre-print servers as they might be prone to bias.

\section{Selection of studies and data extraction}


We imported the search items into a commercially available reference manager for deduplication. Following deduplication, two authors (JN and ZL) screened the titles and abstracts for relevant studies. After screening, and obtaining the full-text manuscript of relevant studies, the same two authors reviewed them carefully for inclusion into our systematic review and meta-analysis. Disagreements that occurred during abstract and title screening, or full-text review were resolved by consensus after discussion with a third author (AC). An author (JN) extracted relevant data from the included studies, and another author (AC) verified the accuracy of the extracted data. Only ICU-specific data were extracted. We extracted the following variables from the included studies: study first author, study location, study period, study type, study population, study sample size, demographical information (age, gender, body-mass index), comorbidities (diabetes mellitus, hypertension, active malignancy, previous venous thromboembolism), laboratory parameters on admission to ICU (platelet count, D-dimer levels), venous thromboembolism prophylaxis regimes, proportion of patients on prophylactic or therapeutic anticoagulation, indication for performing PTE imaging, the incidence of PTE and follow-up period.

\section{Study outcome}

The primary outcome of this study is to estimate and report the weighted average incidence of PTE in critically ill COVID-19 patients that were admitted to the ICU. We considered a positive diagnosis of PTE only if the diagnosis was confirmed by contrast-enhanced computed tomographic imaging of the chest. The secondary outcome of this study is to assess for moderators that could potentially affect the primary outcome.

\section{Risk of bias assessment}

Two authors (JN and ZL) assessed the risk of bias of all included studies by using the ROBINS-I tool.[10] Disagreements were resolved by consensus after discussion with a third author (AC). The ROBINS-I tool was designed specifically to assess the risk of bias in non-randomised studies in seven domains - bias due to confounding, selection bias, bias in classification of interventions, bias due to deviations from intended interventions, bias due to missing data, bias in measurement of outcomes, and bias in selection of reported results. Each included study would be appraised based on the ROBINS-I tool to deduce the overall risk of bias.

\section{Data analysis}

We performed statistical analysis using the meta and metafor packages with R 3.6.3 (R Foundation for Statistical Computing, Vienna, Austria). A frequentist approach was utilized. Meta-analysis of proportions was performed using a random-effects model (DerSimonian and Laird) with logit transformation of observed proportions. The primary outcome was reported as proportions with their respective $95 \%$ confidence intervals (Cl). We assessed statistical heterogeneity using the Cochran's Q test and $\mathrm{I}^{2}$ statistic. In Cochran's Q test, we used a P value of less than 0.1 to represent significant heterogeneity of intervention effects. For the $\mathrm{I}^{2}$ statistic, a value of more than $50 \%$ represented substantial statistical heterogeneity. We performed sensitivity analyses if appropriate. We also performed meta-regression analysis to identify possible moderators that might contribute to statistical heterogeneity. For purposes of the meta-regression, we converted median and interquartile range values to mean and standard deviation using a validated method.[11] We evaluated publication bias with a funnel plot and rank correlation test.

\section{Results}

\section{Study selection}

A thorough and systematic search was conducted according to the pre-defined search protocol as specified in the methods section of this manuscript (Fig. 1). The search yielded a total of 2246 studies, of which 1537 studies remained after deduplication. Following title and abstract screening, we identified 23 studies for full-text review. After completion of full-text review, we included 14 studies into this systematic review and meta-analysis.[12-25]

\section{Risk of bias assessment}

Risk of bias was assessed by using the ROBINS-I tool (Table 1).[10] A single study (Fraissé et al.) was assessed to have a low risk of bias across all domains, and hence deemed to have a low overall risk of bias.[14] Nine studies were considered to have a moderate overall risk of bias, as one or more domains were deemed to be at moderate risk. $[13,16,17,19-22,24,25]$ Four studies were considered to have serious overall risk of bias due to the presence of missing data such as patient comorbidities and ICU characteristics.[12, 15, 18, 23]

\section{Characteristics of included studies}

We included 14 studies with a total of 1182 patients into this systematic review and meta-analysis.[12-25] A summary of study characteristics can be seen in Table 2, whereas a summary of patient characteristics can be seen in Table 3. Four studies were conducted in France (Fraissé et al., Helms et al., Llitjos et al. and Poissy et al.), three in the Netherlands (Beun et al., Klok et al. and Middeldorp et al.), two in Italy (Lodigiani et al. and Tavazzi et al.), two in the United Kingdom (Desborough et al. and Thomas et al.), two in the United States of America (Hippensteel et al. and Maatman et al.), and one in Switzerland (Grandmaison et al.). All studies were conducted between February 2020 and April 2020. Only five studies had reported the duration of follow-up, which varied from seven to 28 days. $[13,16,18,22,25]$ 
Table 2

Summary of study characteristics

\begin{tabular}{|c|c|c|c|c|c|c|c|c|c|}
\hline Study & $\begin{array}{l}\text { Study } \\
\text { location }\end{array}$ & $\begin{array}{l}\text { Study } \\
\text { period }\end{array}$ & $\begin{array}{l}\text { Indication } \\
\text { for ICU } \\
\text { admission }\end{array}$ & $\begin{array}{l}\mathrm{N} \\
(\mathrm{ICU})\end{array}$ & $\begin{array}{l}\text { Prophylactic } \\
\text { anticoagulation } \\
\text { agent }\end{array}$ & $\begin{array}{l}\text { Patients } \\
\text { receiving } \\
\text { therapeutic } \\
\text { anticoagulation } \\
\text { on ICU } \\
\text { admission (\%) }\end{array}$ & $\begin{array}{l}\text { Patients } \\
\text { receiving } \\
\text { prophylactic } \\
\text { anticoagulation } \\
\text { on ICU } \\
\text { admission (\%) }\end{array}$ & $\begin{array}{l}\text { Patients } \\
\text { receiving at } \\
\text { least } \\
\text { anticoagulation } \\
\text { on ICU } \\
\text { admission (\%) }\end{array}$ & $\begin{array}{l}\text { Imaging } \\
\text { Modality } \\
\text { for PTE } \\
\text { diagnosis }\end{array}$ \\
\hline $\begin{array}{l}\text { Beun et al. } \\
\text { [12] }\end{array}$ & Netherlands & $\begin{array}{l}16 \\
\text { March - } \\
9 \text { April }\end{array}$ & NR & 75 & NR & NR & NR & NR & CT scan \\
\hline $\begin{array}{l}\text { Desborough } \\
\text { et al.[13] }\end{array}$ & $\begin{array}{l}\text { United } \\
\text { Kingdom }\end{array}$ & $\begin{array}{l}1 \text { March } \\
-31 \\
\text { March }\end{array}$ & NR & 66 & Dalteparin & 16.7 & 83.3 & 100 & CT scan \\
\hline $\begin{array}{l}\text { Fraissé et al. } \\
\text { [14] }\end{array}$ & France & $\begin{array}{l}6 \text { March } \\
-22 \\
\text { April }\end{array}$ & $\begin{array}{l}\text { Respiratory } \\
\text { failure }\end{array}$ & 92 & NR & 53.3 & 46.7 & 100 & CT scan \\
\hline $\begin{array}{l}\text { Grandmaison } \\
\text { et al.[15] }\end{array}$ & Switzerland & NR & NR & 29 & $\begin{array}{l}\text { Enoxaparin, } \\
\text { UFH }\end{array}$ & 3.4 & 89.7 & 93.1 & CT scan \\
\hline $\begin{array}{l}\text { Helms et al. } \\
\text { [16] }\end{array}$ & France & $\begin{array}{l}3 \text { March } \\
-31 \\
\text { March }\end{array}$ & $\begin{array}{l}\text { Acute } \\
\text { respiratory } \\
\text { distress } \\
\text { syndrome } \\
\text { based on } \\
\text { Berlin } \\
\text { definition }\end{array}$ & 150 & LMWH, UFH & 30 & 70 & 100 & CT scan \\
\hline $\begin{array}{l}\text { Hippensteel } \\
\text { et al.[17] }\end{array}$ & $\begin{array}{l}\text { United } \\
\text { States of } \\
\text { America }\end{array}$ & $\begin{array}{l}18 \\
\text { March - } \\
14 \text { April }\end{array}$ & NR & 91 & NR & NR & NR & NR & CT scan \\
\hline Klok et al.[18] & Netherlands & $\begin{array}{l}7 \text { March } \\
-5 \text { April }\end{array}$ & NR & 184 & Nadroparin & 9.2 & 90.8 & 100 & CT scan \\
\hline $\begin{array}{l}\text { Llitjos et al. } \\
\text { [19] }\end{array}$ & France & $\begin{array}{l}19 \\
\text { March - } \\
11 \text { April }\end{array}$ & $\begin{array}{l}\text { Respiratory } \\
\text { failure }\end{array}$ & 26 & LMWH, UFH & 69.2 & 30.8 & 100 & CT scan \\
\hline $\begin{array}{l}\text { Lodigiani et } \\
\text { al.[20] }\end{array}$ & Italy & $\begin{array}{l}13 \\
\text { February } \\
-10 \\
\text { April }\end{array}$ & NR & 61 & LMWH & 3.3 & 96.7 & 100 & CT scan \\
\hline $\begin{array}{l}\text { Maatman et } \\
\text { al.[21] }\end{array}$ & $\begin{array}{l}\text { United } \\
\text { States of } \\
\text { America }\end{array}$ & $\begin{array}{l}12 \\
\text { March - } \\
31 \\
\text { March }\end{array}$ & $\begin{array}{l}\mathrm{SpO}_{2} \leq \\
94 \%, \mathrm{RR} \geq \\
30 \\
\mathrm{PaO}_{2} / \mathrm{FiO}_{2} \\
\text { ratio } \leq \\
300 \text { mmHg, } \\
\text { or requiring } \\
\text { mechanical } \\
\text { ventilation }\end{array}$ & 109 & $\begin{array}{l}\text { Enoxaparin, } \\
\text { UFH }\end{array}$ & 6.4 & 93.6 & 100 & CT scan \\
\hline $\begin{array}{l}\text { Middeldorp } \\
\text { et al.[22] }\end{array}$ & Netherlands & $\begin{array}{l}2 \text { March } \\
-12 \\
\text { April }\end{array}$ & NR & 75 & Nadroparin & 9.3 & 90.7 & 100 & CT scan \\
\hline $\begin{array}{l}\text { Poissy et al. } \\
\text { [23] }\end{array}$ & France & $\begin{array}{l}27 \\
\text { February } \\
-31 \\
\text { March }\end{array}$ & NR & 107 & LMWH, UFH & NR & NR & NR & CT scan \\
\hline $\begin{array}{l}\text { Tavazzi et al. } \\
\text { [24] }\end{array}$ & Italy & NR & NR & 54 & LMWH & 0 & 100 & 100 & CT scan \\
\hline $\begin{array}{l}\text { Thomas et } \\
\text { al.[25] }\end{array}$ & $\begin{array}{l}\text { United } \\
\text { Kingdom }\end{array}$ & $\begin{array}{l}15 \\
\text { March - } \\
14 \text { April }\end{array}$ & NR & 63 & Dalteparin & 0 & 100 & 100 & CT scan \\
\hline
\end{tabular}

ICU intensive care unit, BMI body-mass index, PTE pulmonary thromboembolism, CT computed tomographic, NR not reported, LMWH low molecular weight h respiratory rate 
Table 3

Summary of patient characteristics

\begin{tabular}{|c|c|c|c|c|c|c|c|c|c|c|c|c|}
\hline Study & $\begin{array}{l}\text { Age } \\
\text { (years) }\end{array}$ & $\begin{array}{l}\text { Male } \\
(\%)\end{array}$ & $\begin{array}{l}\text { BMI } \\
\left(\mathrm{kg} / \mathrm{m}^{2}\right)\end{array}$ & $\begin{array}{l}\text { DM } \\
(\%)\end{array}$ & $\begin{array}{l}\text { Hypertension } \\
\text { (\%) }\end{array}$ & $\begin{array}{l}\text { Malignancy } \\
(\%)\end{array}$ & $\begin{array}{l}\text { Previous } \\
\text { VTE (\%) }\end{array}$ & $\begin{array}{l}\text { Platelet } \\
\text { count } \\
\left(\times 10^{9} / \mathrm{L}\right)\end{array}$ & $\begin{array}{l}\text { D- } \\
\text { dimer } \\
\text { (mg/L) }\end{array}$ & $\begin{array}{l}\text { Patients } \\
\text { intubated } \\
\text { (\%) }\end{array}$ & $\begin{array}{l}\text { Patients } \\
\text { on } \\
\text { inotropes } \\
(\%)\end{array}$ & $\begin{array}{l}\text { Patients } \\
\text { on RRT } \\
\text { (\%) }\end{array}$ \\
\hline $\begin{array}{l}\text { Beun et al. } \\
\text { [12] }\end{array}$ & NR & NR & NR & NR & NR & NR & NR & NR & NR & NR & NR & NR \\
\hline \multirow{2}{*}{$\begin{array}{l}\text { Desborough } \\
\text { et al.[13] }\end{array}$} & 59 & 72.7 & 28 & 40.9 & 45.5 & 7.6 & 7.6 & 207 & 2.4 & 78.8 & 47 & 27.3 \\
\hline & $\begin{array}{l}(49- \\
66)\end{array}$ & & $\begin{array}{l}(24- \\
34)\end{array}$ & & & & & $\begin{array}{l}(154- \\
272)\end{array}$ & $\begin{array}{l}(1.1- \\
6.2)\end{array}$ & & & \\
\hline \multirow{2}{*}{$\begin{array}{l}\text { Fraissé et al. } \\
\text { [14] }\end{array}$} & 61 & 79.3 & 30 & 38 & 64.1 & NR & 5.4 & 227 & 2.4 & 89.1 & 62 & 23.9 \\
\hline & $\begin{array}{l}(55- \\
70)\end{array}$ & & $\begin{array}{l}(26- \\
35)\end{array}$ & & & & & $\begin{array}{l}(182- \\
307)\end{array}$ & $\begin{array}{l}(1.7- \\
7.9)\end{array}$ & & & \\
\hline $\begin{array}{l}\text { Grandmaison } \\
\text { et al.[15] }\end{array}$ & NR & 72.1 & NR & NR & NR & 6.9 & 6.9 & NR & NR & NR & NR & NR \\
\hline \multirow{2}{*}{$\begin{array}{l}\text { Helms et al. } \\
\text { [16] }\end{array}$} & 63 & 81.3 & NR & 20 & NR & 6 & 5.3 & 200 & 2.3 & 100 & NR & NR \\
\hline & $\begin{array}{l}(53- \\
71)\end{array}$ & & & & & & & $\begin{array}{l}(152- \\
267)\end{array}$ & $\begin{array}{l}(1.2- \\
20)\end{array}$ & & & \\
\hline $\begin{array}{l}\text { Hippensteel } \\
\text { et al.[17] }\end{array}$ & $\begin{array}{l}56 \pm \\
16\end{array}$ & 58.2 & $\begin{array}{l}32.4 \pm \\
9.9\end{array}$ & 30.8 & NR & 3.3 & NR & $200 \pm 91$ & $\begin{array}{l}3.0 \pm \\
10.3\end{array}$ & 84.6 & 67 & NR \\
\hline Klok et al.[18] & $\begin{array}{l}64 \pm \\
12\end{array}$ & 75.5 & NR & NR & NR & 2.7 & NR & NR & NR & NR & NR & 12.5 \\
\hline \multirow{2}{*}{$\begin{array}{l}\text { Llitjos et al. } \\
\text { [19] }\end{array}$} & 68 & 76.9 & 30.2 & NR & 84.6 & 0 & 3.8 & 234 & 1.8 & 100 & 88.5 & 15.4 \\
\hline & $\begin{array}{l}(52- \\
75)\end{array}$ & & $\begin{array}{l}(25.5- \\
33.5)\end{array}$ & & & & & $\begin{array}{l}(169- \\
306)\end{array}$ & $\begin{array}{l}(1.1- \\
2.9)\end{array}$ & & & \\
\hline \multirow{2}{*}{$\begin{array}{l}\text { Lodigiani et } \\
\text { al.[20] }\end{array}$} & 61 & 80.3 & NR & 18 & 42.6 & 3.3 & 0 & NR & NR & NR & NR & NR \\
\hline & $\begin{array}{l}(55- \\
69)\end{array}$ & & & & & & & & & & & \\
\hline \multirow{2}{*}{$\begin{array}{l}\text { Maatman et } \\
\text { al.[21] }\end{array}$} & \multirow{2}{*}{$\begin{array}{l}61 \pm \\
16\end{array}$} & \multirow[t]{2}{*}{56.9} & \multirow{2}{*}{$\begin{array}{l}34.8 \pm \\
11.8\end{array}$} & \multirow[t]{2}{*}{39.4} & \multirow[t]{2}{*}{67.9} & \multirow[t]{2}{*}{ NR } & \multirow[t]{2}{*}{ NR } & 207 & 0.5 & 94.5 & 64.2 & 14.7 \\
\hline & & & & & & & & $\begin{array}{l}(152- \\
255)\end{array}$ & $\begin{array}{l}(0.3- \\
1.0)\end{array}$ & & & \\
\hline \multirow{2}{*}{$\begin{array}{l}\text { Middeldorp } \\
\text { et al.[22] }\end{array}$} & \multirow{2}{*}{$\begin{array}{l}62 \pm \\
10\end{array}$} & \multirow[t]{2}{*}{77.3} & 27 & \multirow[t]{2}{*}{ NR } & \multirow[t]{2}{*}{ NR } & \multirow[t]{2}{*}{4} & \multirow[t]{2}{*}{2.7} & \multirow[t]{2}{*}{$251 \pm 89$} & 2 & NR & NR & NR \\
\hline & & & $\begin{array}{l}(24- \\
29)\end{array}$ & & & & & & $\begin{array}{l}(0.8- \\
8.1)\end{array}$ & & & \\
\hline $\begin{array}{l}\text { Poissy et al. } \\
\text { [23] }\end{array}$ & NR & NR & NR & NR & NR & NR & NR & NR & NR & NR & NR & NR \\
\hline $\begin{array}{l}\text { Tavazzi et al. } \\
\text { [24] }\end{array}$ & $68 \pm 7$ & 83.3 & $\begin{array}{l}29.3 \pm \\
4.4\end{array}$ & NR & NR & NR & NR & NR & NR & 100 & NR & NR \\
\hline \multirow{2}{*}{$\begin{array}{l}\text { Thomas et } \\
\text { al.[25] }\end{array}$} & \multirow[t]{2}{*}{ NR } & \multirow[t]{2}{*}{69.8} & \multirow[t]{2}{*}{$N R$} & \multirow[t]{2}{*}{ NR } & \multirow[t]{2}{*}{ NR } & \multirow[t]{2}{*}{1.6} & \multirow[t]{2}{*}{1.6} & \multirow[t]{2}{*}{ NR } & $3.9 *$ & \multirow[t]{2}{*}{82.5} & NR & 36.5 \\
\hline & & & & & & & & & $\begin{array}{l}(1.2- \\
36.3)\end{array}$ & & & \\
\hline
\end{tabular}

BMI body-mass index, DM diabetes mellitus, CKD chronic kidney disease, VTE venous thromboembolism, RRT renal replacement therapy, ECMO extracorpore membrane oxygenation, NR not reported

Unless otherwise stated, all values are represented in percentages (\%), mean \pm standard deviation, or median (interquartile range)

*Value represented as median (range)

\section{Indication for ICU admission}

Only four studies had reported their indication for ICU admission.[14, 16, 19, 21] Two studies (Fraissé et al., and Llitjos et al.) defined their ICU admission criteria as any patient with respiratory failure.[14,19] Helms et al. defined their ICU admission criteria as patients who have acute respiratory distress syndrome based on the Berlin 2012 definition, whereas the study by Maatman et al. defined their ICU admission criteria as any patient with an oxygen saturation of $94 \%$ or less, respiratory rate of 30 breaths per minute or more, $\mathrm{PaO}_{2} / \mathrm{FiO}_{2}$ ratio of $300 \mathrm{mmHg}$ or less, or if requiring mechanical ventilation.[16, 21]

Prophylactic anticoagulation regime and compliance

Eleven studies reported the use of either low-molecular-weight heparin (enoxaparin, nadroparin, dalteparin or unspecified) or unfractionated heparin for venous thromboembolism prophylaxis in varying doses.[13,15, 16, 18-25] The majority of studies had also reported information on the proportion of patients receiving therapeutic or prophylactic anticoagulation in ICU. The proportion of patients that was started on therapeutic anticoagulation in ICU varied from $0-$ $69.2 \%$, whilst the proportion of patients that was started on prophylactic anticoagulation varied from $30.8-100 \%$. Overall, in ten out of the 11 studies that had 
sufficient information on anticoagulation practices, $100 \%$ of patients received at least prophylactic anticoagulation. [13, 14, 16, 18-22, 24, 25] In the study by Grandmaison et al., $93.1 \%$ of patients received at least prophylactic anticoagulation.[15]

\section{Modality and indication for pulmonary thromboembolism imaging}

Contrast-enhanced computed tomographic scan was the principal modality used to diagnose PTE in all included studies.[12-25] Eight studies specifically reported the indication for performing PTE imaging. [14-16, 19, 20, 22, 23, 25] All eight studies adopted a selective approach based on the patient's clinical condition to decide if PTE imaging was required. In these studies, PTE imaging was only performed if there was a clinical suspicion of PTE, for example, if patients had persistent respiratory failure, deteriorating respiratory function or haemodynamic status, or if there was a rapid increase in D-dimer levels.

\section{Primary outcome: Incidence of pulmonary thromboembolism}

The reported incidence of PTE ranged from 3.3-26.7\%. Including all 14 studies, the weighted average incidence of PTE in COVID-19 patients after admission to the intensive care unit was $11.09 \%$ (95\% Cl 7.72-15.69\%, $\mathrm{I}^{2}=78 \%$, Cochran's $\mathrm{Q}$ test $\left.\mathrm{P}<0.01\right)$ after random-effects meta-analysis of proportions (Fig. 2).[1225] Significant statistical heterogeneity was present as evidenced by high $I^{2}$ value and a Cochran's $Q$ test $P$ value of less than 0.1 .

\section{Meta-regression and moderator assessment}

Meta-regression with a mixed-effects model was performed to examine if the observed heterogeneity could be contributed by possible moderators such as patient or study characteristics (Table 4). Univariate meta-regression revealed that the proportion of male patients, platelet count on admission to ICU, and proportion of patients on therapeutic anticoagulation were possible significant moderators. These three significant moderators were added into the multivariable meta-regression model for further analysis. Multivariable meta-regression revealed that the proportion of male patients remained as the only significant moderator in this meta-analysis. A higher proportion of males was associated with a higher incidence of PTE.

Table 4

Meta-regression analysis

\begin{tabular}{|c|c|c|c|c|c|c|c|c|}
\hline \multirow[b]{2}{*}{ Variables } & \multicolumn{4}{|c|}{ Univariate analysis } & \multicolumn{4}{|c|}{ Multivariate analysis } \\
\hline & Coeff & SE & $95 \% \mathrm{Cl}$ & $\mathbf{P}$ & Coeff & SE & $95 \% \mathrm{Cl}$ & $\mathbf{P}$ \\
\hline Sample size & 0.00 & 0.00 & $-0.01-0.01$ & 0.55 & - & E & - & - \\
\hline Age (years) & 0.06 & 0.08 & $-0.09-0.22$ & 0.40 & F & - & - & - \\
\hline Male gender (\%) & 0.06 & 0.02 & $0.03-0.09$ & $<0.01$ & 0.05 & 0.02 & $0.00-0.09$ & 0.03 \\
\hline Body-mass index $\left(\mathrm{kg} / \mathrm{m}^{2}\right)$ & -0.14 & 0.12 & $-0.37-0.10$ & 0.25 & + & 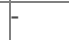 & 1 & 1 \\
\hline Diabetes mellitus (\%) & 0.00 & 0.04 & $-0.08-0.08$ & 0.95 & - & - & - & - \\
\hline Hypertension (\%) & 0.04 & 0.03 & $-0.01-0.09$ & 0.15 & - & - & F & F \\
\hline Active malignancy (\%) & -0.06 & 0.09 & $-0.24-0.13$ & 0.54 & - & F & F & F \\
\hline Previous VŤE (\%) & 0.08 & 0.11 & $-0.13-0.28$ & 0.48 & - & - & - & - \\
\hline Platelet count & 0.03 & 0.01 & $0.01-0.05$ & 0.01 & 0.01 & 0.01 & $-0.01-0.02$ & 0.42 \\
\hline D-dimer level & 0.00 & 0.00 & $-0.00-0.01$ & 0.44 & - & - & - & - \\
\hline Patients on therapeutic anticoagulation (\%) & 0.02 & 0.01 & $0.01-0.03$ & $<0.01$ & 0.01 & 0.01 & $-0.01-0.03$ & 0.24 \\
\hline Patients intubated (\%) & 0.03 & 0.04 & $-0.04-0.10$ & 0.43 & - & - & - & - \\
\hline Patients on inotropes (\%) & 0.03 & 0.03 & $-0.03-0.09$ & 0.36 & - & F & F & 5 \\
\hline Patients on RRT (\%) & -0.02 & 0.04 & $-0.09-0.06$ & 0.66 & - & - & - & - \\
\hline Patients on ECMO (\%) & 0.07 & 0.15 & $-0.21-0.35$ & 0.63 & $F$ & $F$ & $F$ & $F$ \\
\hline
\end{tabular}

error VTE venous thromboembolism

\section{Publication bias}

We assessed publication bias by using a funnel plot and the rank correlation test. The funnel plot of all included studies is as shown in Fig. 3. The rank correlation test proved that there was no significant funnel plot asymmetry. $(P=0.19)$.

\section{Discussion}

In this meta-analysis, we found that the weighted average incidence of PTE in critically ill COVID-19 patients after admission to the ICU to be $11.09 \%$ (95\% CI 7.72-15.69\%). This is the first meta-analysis performed to elucidate specifically the incidence of PTE in critically ill COVID-19 patients. There have been other meta-analyses that looked at the incidence of venous thromboembolism as a whole, but made no effort to segregate specifically the incidence of PTE.[26] The results from this meta-analysis can be used to plan healthcare resources, to educate patients and healthcare workers, and to plan for future studies (i.e. sample size calculation) about PTE in COVID-19 patients.

We also identified the proportion of males to be a significant moderator and significant source of statistical heterogeneity in the incidence of PTE after univariate and multivariate meta-regression analysis. Our analysis shows that studies that had a higher proportion of male patients had a higher incidence of reported PTE. This observed phenomenon can be corroborated by several studies that had been performed in the past that have demonstrated a higher risk of first episode or recurrent venous thromboembolism in males compared to females.[27] Several hypotheses such as genetic variations or differences in environmental factors have been suggested to account for the differential risks of venous thromboembolism, but the evidence is still unclear.[28]

The significant statistical heterogeneity of our primary outcome - the incidence of PTE could also be explained by other moderators that were not included in our meta-regression analysis. One possibility that cannot be easily analysed or accounted for would be how study location and study period can affect the incidence of PTE. Studies by Lodigiani et al. and Tavazzi et al., which were both conducted in Italy, had reported relatively low PTE incidences of $3.3 \%$ and $3.7 \%$ respectively as compared to studies conducted outside of Italy.[20,24] We postulate several possible reasons for the observed geographical disparity in reported PTE incidences. First, Lodigiani et al. had reported that only $13.1 \%$ of their study population had PE imaging performed.[20] Compared to other studies included in this meta-analysis, this seemed to be considerably lower. As such, PTE could have been underdiagnosed. On the contrary, other studies with more widespread PTE imaging may have diagnosed a larger number of patients with subclinical or asymptomatic PTE. Several retrospective studies reviewed CT scans performed COVID-19 patients regardless of clinical context had reported radiological findings of PTE in up to 50\% of patients.[29, 30] Undoubtedly, most PTE findings in these radiological studies may be clinically insignificant. Furthermore, resource constraints secondary to the COVID-19 pandemic might contributed to the possible geographical disparity in PTE incidence. The studies by Lodigiani et al. and Tavazzi et al. were conducted in the Lombardy region of Italy, which had the most number of COVID-19 cases and the highest case fatality rate in Italy.[31] As such, the institutions at which the 
studies were conducted might have faced possible resource constraints that may have inevitably led to a more selective or conservative approach to PTE imaging and diagnosis.[32] Lastly, other moderators such as follow-up duration might have also contributed to the statistical heterogeneity, but cannot be evaluated properly due to missing data.

Our study also provides some insight into the various prophylactic anticoagulation regimes adopted by different institutions. Interestingly, two studies conducted in the Netherlands had doubled the doses of their anticoagulation regimes around late March and early April. Although the authors did not clarify the reasons why, it is most likely due to an increased awareness of the thromboembolic manifestations of COVID-19. Ten studies had also reported that $100 \%$ of their study population had received at least prophylactic anticoagulation. However, despite a high degree of compliance to anticoagulation, a considerable proportion of critically-ill COVID-19 patients still developed PTE. Due to this phenomenon, several studies have suggested an intermediate dose or therapeutic anticoagulation regime for this group of patients.[33,34] Several institutions around the world are currently planning, or have begun recruiting for clinical trials to evaluate the effects of higher dose anticoagulation for critically-ill COVID-19 patients.[35]

As more evidence emerges, we hope to understand more about the hypercoagulable state that COVID-19 is associated with. Some studies have demonstrated that critically-ill COVID-19 patients may exhibit deranged haemostatic parameters such as thrombocytopenia, elevated D-dimer levels and prolongation of prothrombin time.[36] Other studies have also shown that a large proportion of critically-ill COVID-19 have a positive lupus anticoagulant test and hypercoagulable thromboelastometry profiles.[37, 38] Based on these studies, it is undeniable that severe COVID-19 infection is associated with an abnormal haemostatic state. However, whether or not COVID-19 has a direct effect on coagulation pathways, or whether these abnormalities are secondary to the cytokine storm or systemic inflammatory response that can be associated with any severe insult to the body remains to be determined.[39]

There are several limitations to our meta-analysis. First, there are differences in study population that we cannot account for. Many of the included studies did not report essential information such as the indication for ICU admission, patient comorbidities and follow-up duration. Due to significant missing data, four out of the 14 included studies were deemed to be at high overall risk of bias. Moreover, we were also unable to assess the effect of PTE incidence on mortality, as most studies did not report mortality outcomes.

\section{Conclusion}

In conclusion, the weighted average incidence of PTE in critically-ill COVID-19 patients admitted to the ICU for treatment was $11.09 \%$, despite the fact that almost all patients received least prophylactic doses of anticoagulation therapy. Although there was significant heterogeneity in our meta-analysis, we identified the proportion of male patients as a significant moderator and contributor of heterogeneity. Clinicians should be aware that PTE can occur in a significant proportion of COVID-19 patients receiving ICU care despite adequate prophylactic anticoagulation, and should investigate further should clinical suspicion arises. Moving ahead, more studies are also needed to determine the optimal anticoagulation strategy in critically ill COVID-19 patients.

\section{Declarations}

\section{Take home message}

The risk of developing PTE is high in COVID-19 patients that are treated in the ICU setting, despite receiving prophylactic anticoagulation. Clinicians should investigate further promptly if clinical suspicion arises. More studies are needed to elucidate this thromboembolic phenomenon and determine the optimal anticoagulation strategy.

\section{Funding}

The authors declare no source of funding was utilized in this study.

\section{Conflicts of interest}

The authors declare no conflicts of interest.

\section{Availability of data and materials}

The datasets used and/or analysed during the current study are available from the corresponding author (JN) on reasonable request.

\section{Ethics approval}

Ethics approval was not required for this study as it does not contain any form of individual patient data.

\section{Consent to participate}

Not applicable

\section{Consent for publication}

All authors consent for publication of our submitted work.

\section{Author's contribution}


Jun Jie $\mathrm{Ng}(\mathrm{JN})$ and Zhen Chang Liang (ZL) contributed both contributed equally to the conception, design of this study; acquisition, analysis and interpretation of data. JN drafted the initial manuscript. Andrew MTL Choong (AC) contributed to the design of this study; acquisition and interpretation of data; and critically revised the manuscript. All authors have approved the submitted version of this manuscript. The corresponding author (JN) attests that all listed authors meet authorship criteria and that no others meeting the criteria have been omitted. JN is responsible for the overall content of this manuscript as guarantor.

\section{References}

1. World Health Organization. COVID-19 situation reports. 2020, https://www.who.int/emergencies/diseases/novel-coronavirus-2019/situation-reports/. Accessed 1 September 2020.

2. Castelli R, Gidaro A. Abnormal Hemostatic Parameters and Risk of Thromboembolism Among Patients With COVID-19 Infection. J Hematol. 2020 Apr;9(12):1-4.

3. Yao XH, Li TY, He ZC, et al. [A pathological report of three COVID-19 cases by minimally invasive autopsies]. Zhonghua Bing Li Xue Za Zhi. [published online: March 15, 2020]. 10.3760/cma.j.cn112151-20200312-00193

4. Han H, Yang L, Liu R, et al. Prominent changes in blood coagulation of patients with SARS-CoV-2 infection. Clin Chem Lab Med. [published online: March 16, 2020]. 10.1515/cclm-2020-0188

5. Tang N, Li D, Wang X, Sun Z. Abnormal coagulation parameters are associated with poor prognosis in patients with novel coronavirus pneumonia. J Thromb Haemost. 2020 04;18(4):844-7.

6. Poggiali E, Bastoni D, loannilli E, Vercelli A, Magnacavallo A. Deep Vein Thrombosis and Pulmonary Embolism: Two Complications of COVID-19 Pneumonia. Eur J Case Rep Intern Med. 2020;7(5):001646.

7. Cochrane Training: Cochrane Handbook for Systematic Reviews of Interventions. 2020, https://training.cochrane.org/handbook. Accessed 10 May 2020.

8. Moher D, Liberati A, Tetzlaff J, et al. Preferred reporting items for systematic reviews and meta-analyses: the PRISMA statement. BMJ. 2009 Jul 21;339:b2535.

9. World Health Organization: WHO Timeline - COVID-19. 2020, https://www.who.int/news-room/detail/27-04-2020-who-timeline--covid-19. Accessed 1 September 2020.

10. Sterne JA, Hernán MA, Reeves BC, et al. ROBINS-I: a tool for assessing risk of bias in non-randomised studies of interventions. BMJ. 2016 Oct 12;355:i4919.

11. Wan X, Wang W, Liu J, Tong T. Estimating the sample mean and standard deviation from the sample size, median, range and/or interquartile range. BMC Med Res Methodol. 2014 Dec 19;14:135.

12. Beun R, Kusadasi N, Sikma M, Westerink J, Huisman A. Thromboembolic events and apparent heparin resistance in patients infected with SARS-CoV-2. Int J Lab Hematol. 2020 Jun;42 Suppl 1(Suppl 1):19-20.

13. Desborough MJR, Doyle AJ, Griffiths A, Retter A, Breen KA, Hunt BJ. Image-proven thromboembolism in patients with severe COVID-19 in a tertiary critical care unit in the United Kingdom. Thromb Res. 2020 May 29;193:1-4.

14. Fraissé M, Logre E, Pajot O, Mentec H, Plantefève G, Contou D. Thrombotic and hemorrhagic events in critically ill COVID-19 patients: a French monocenter retrospective study. Crit Care. 2020 Jun 2;24(1):275.

15. Grandmaison G, Andrey A, Périard D, et al. Systematic Screening for Venous Thromboembolic Events in COVID-19 Pneumonia. TH Open. 2020 Jun 8;4(2):e113-e115.

16. Helms J, Tacquard C, Severac F, et al. High risk of thrombosis in patients with severe SARS-CoV-2 infection: a multicenter prospective cohort study. Intensive Care Med. [published online: May 04, 2020]. 10.1007/s00134-020-06062-x

17. Hippensteel JA, Burnham EL, Jolley SE. Prevalence of venous thromboembolism in critically ill patients with COVID-19. Br J Haematol. 2020 Aug;190(3):e134-e137.

18. Klok FA, Kruip MJHA, van der Meer NJM, et al. Incidence of thrombotic complications in critically ill ICU patients with COVID-19. Thromb Res. [published online: April 10, 2020]. 10.1016/j.thromres.2020.04.013

19. Llitjos JF, Leclerc M, Chochois C, et al. High incidence of venous thromboembolic events in anticoagulated severe COVID-19 patients. J Thromb Haemost. [published online: April 22, 2020]. 10.1111/jth.14869

20. Lodigiani C, lapichino G, Carenzo L, et al. Venous and arterial thromboembolic complications in COVID-19 patients admitted to an academic hospital in Milan, Italy. Thromb Res. [published online: April 23, 2020]. 10.1016/j.thromres.2020.04.024

21. Maatman TK, Jalali F, Feizpour C, et al. Routine Venous Thromboembolism Prophylaxis May Be Inadequate in the Hypercoagulable State of Severe Coronavirus Disease 2019. Crit Care Med. 2020 Sep;48(9):e783-e790.

22. Middeldorp S, Coppens M, van Haaps TF, et al. Incidence of venous thromboembolism in hospitalized patients with COVID-19. J Thromb Haemost. [published online: May 05, 2020]. 10.1111/jth.14888

23. Poissy J, Goutay J, Caplan M, et al. Pulmonary Embolism in COVID-19 Patients: Awareness of an Increased Prevalence. Circulation. [published online: April 24, 2020]. 10.1161/CIRCULATIONAHA.120.047430

24. Tavazzi G, Civardi L, Caneva L, Mongodi S, Mojoli F. Thrombotic events in SARS-CoV-2 patients: an urgent call for ultrasound screening. Intensive Care Med. [published online: April 22, 2020]. 10.1007/s00134-020-06040-3 
25. Thomas W, Varley J, Johnston A, et al. Thrombotic complications of patients admitted to intensive care with COVID-19 at a teaching hospital in the United Kingdom. Thromb Res. [published online: April 25, 2020]. 10.1016/j.thromres.2020.04.028

26. Porfidia A, Valeriani E, Pola R, Porreca E, Rutjes AWS, Di Nisio M. Venous thromboembolism in patients with COVID-19: Systematic review and metaanalysis [published online: Aug 12, 2020]. Thromb Res. 2020;196:67-74.

27. Douketis J, Tosetto A, Marcucci M, et al. Risk of recurrence after venous thromboembolism in men and women: patient level meta-analysis. BMJ. 2011 Feb 24;342:d813.

28. Roach, REJ, Cannegieter, SC, Lijfering, WM. Differential risks in men and women for first and recurrent venous thrombosis: the role of genes and environment. J Thromb Haemost 2014; 12: 1593- 1600.

29. Grillet F, Behr J, Calame P, Aubry S, Delabrousse E. Acute Pulmonary Embolism Associated with COVID-19 Pneumonia Detected by Pulmonary CT Angiography. Radiology. [published online: April 23, 2020]. 10.1148/radiol.2020201544

30. Leonard-Lorant I, Delabranche X, Severac F, et al. Acute Pulmonary Embolism in COVID-19 Patients on CT Angiography and Relationship to D-Dimer Levels. Radiology. [published online: April 23, 2020]. 10.1148/radiol.2020201561

31. Sebastiani G, Massa M, Riboli E. Covid-19 epidemic in Italy: evolution, projections and impact of government measures. Eur J Epidemiol. 2020 Apr;35(4):341-5.

32. Grasselli G, Pesenti A, Cecconi M. Critical Care Utilization for the COVID-19 Outbreak in Lombardy, Italy: Early Experience and Forecast During an Emergency Response. JAMA. [published online: March 13, 2020]. 10.1001/jama.2020.4031

33. Song JC, Wang G, Zhang W, Zhang Y, Li WQ, Zhou Z. Chinese expert consensus on diagnosis and treatment of coagulation dysfunction in COVID-19. Mil Med Res. 202004 20;7(1):19.

34. Thachil J, Tang N, Gando S, et al. ISTH interim guidance on recognition and management of coagulopathy in COVID-19. J Thromb Haemost. 2020 05;18(5):1023-6.

35. S. National Library of Medicine ClinicalTrials.gov: Intermediate or Prophylactic-Dose Anticoagulation for Venous or Arterial Thromboembolism in Severe COVID-19 (IMPROVE) (NCT04367831). 2020, https://clinicaltrials.gov/ct2/show/NCT04367831. Accessed 14 May 2020.

36. Bowles L, Platton S, Yartey N, et al. Lupus Anticoagulant and Abnormal Coagulation Tests in Patients with Covid-19. N Engl J Med. [published online: May 05, 2020]. 10.1056/NEJMc2013656

37. Han H, Yang L, Liu R, et al. Prominent changes in blood coagulation of patients with SARS-CoV-2 infection. Clin Chem Lab Med. [published online: March 16, 2020]. 10.1515/cclm-2020-0188

38. Pavoni V, Gianesello L, Pazzi M, Stera C, Meconi T, Frigieri FC. Evaluation of coagulation function by rotation thromboelastometry in critically ill patients with severe COVID-19 pneumonia. J Thromb Thrombolysis. [published online: May 11, 2020]. 10.1007/s11239-020-02130-7

39. Jose RJ, Manuel A. COVID-19 cytokine storm: the interplay between inflammation and coagulation. Lancet Respir Med. [published online: April 27, 2020]. $10.1016 /$ S2213-2600(20)30216-2

\section{Tables}

Due to technical limitations, table 1 is only available as a download in the Supplemental Files section.

\section{Figures}




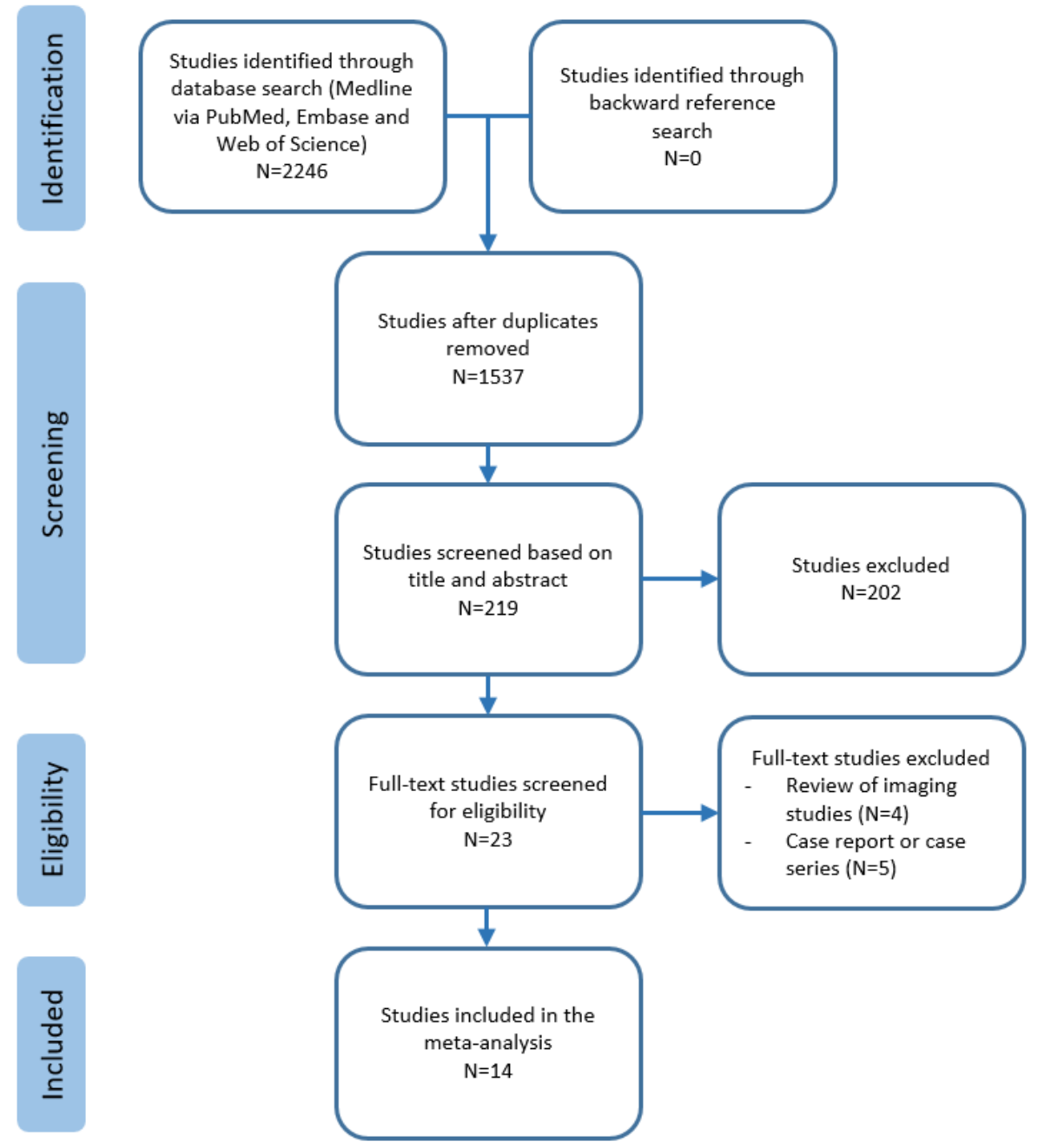

Figure 1

PRISMA flowchart for study selection 


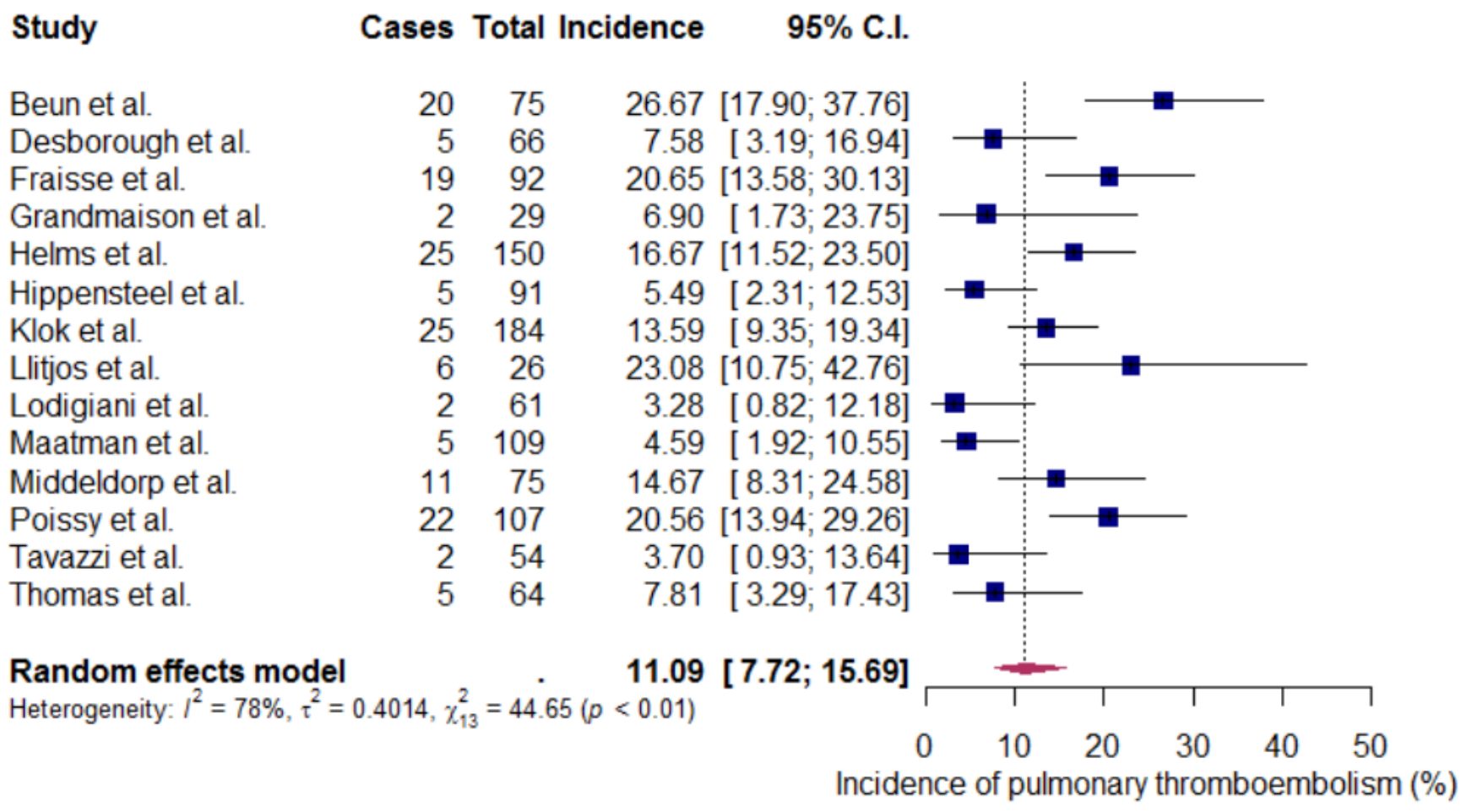

Figure 2

Forest plot showing the weighted average incidence of pulmonary thromboembolism in critically-ill COVID-19 patients admitted to the intensive care unit for treatment 


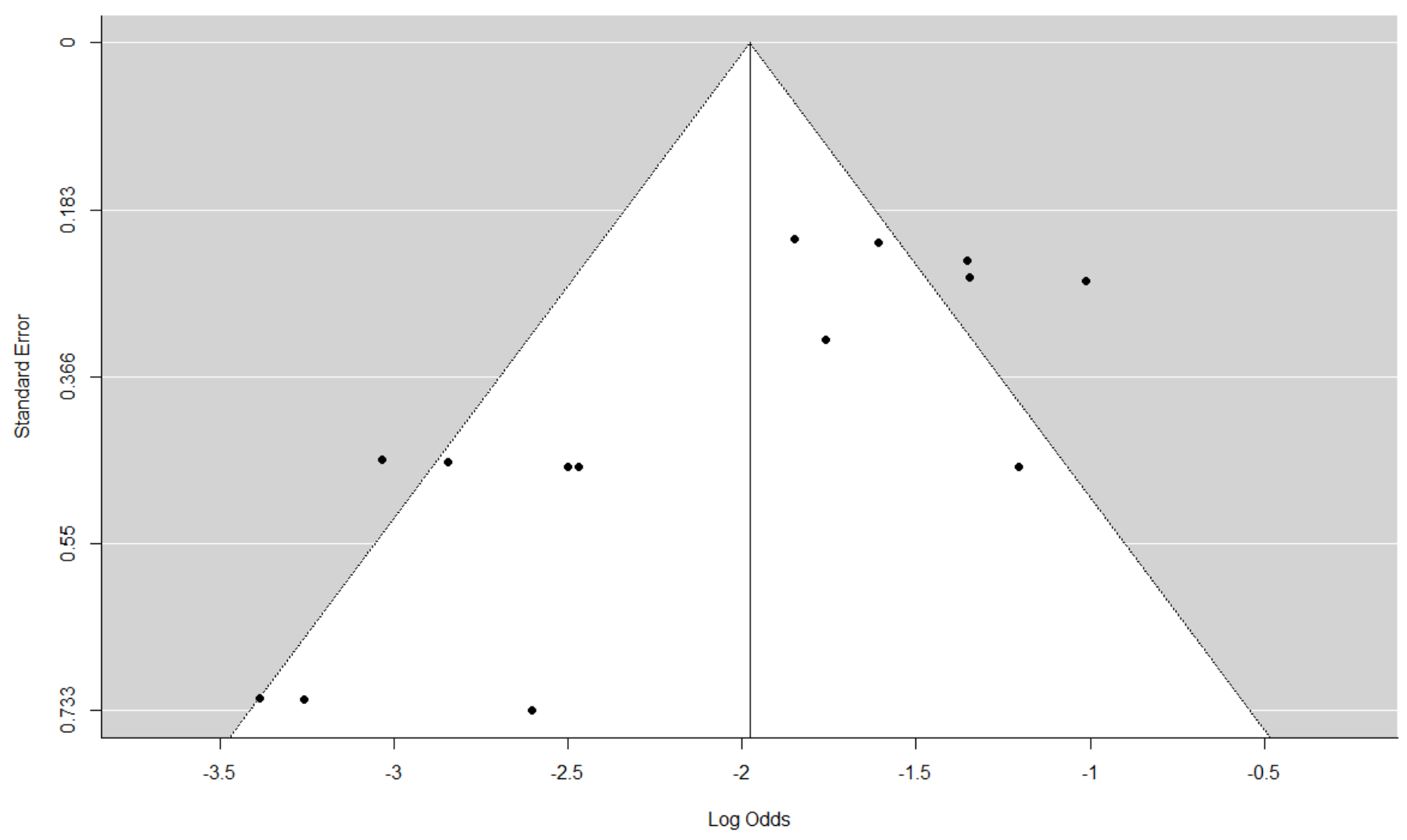

Figure 3

Funnel plot for assessment of publication bias

\section{Supplementary Files}

This is a list of supplementary files associated with this preprint. Click to download.

- Table1ROBINS.docx 